\title{
Dessin d'enfant and a description of mutually nonlocal 7-branes without using branch cuts
}

\author{
Shin Fukuchi, ${ }^{2, *}$ Naoto Kan, ${ }^{2, \dagger}$ Shun’ya Mizoguchi, ${ }^{1,2,}$ and Hitomi Tashiro ${ }^{2,8}$ \\ ${ }^{1}$ Theory Center, Institute of Particle and Nuclear Studies, KEK Tsukuba, Ibaraki, 305-0801, Japan \\ ${ }^{2}$ SOKENDAI (The Graduate University for Advanced Studies) Tsukuba, Ibaraki, 305-0801, Japan
}

(Received 30 July 2019; published 30 December 2019)

\begin{abstract}
We consider the special roles of the zero loci of the Weierstrass invariants $g_{2}(\tau(z))$ and $g_{3}(\tau(z))$ in $\mathrm{F}$ theory on an elliptic fibration over $\mathbb{P}^{1}$ or a further fibration thereof. They are defined as the zero loci of the coefficient functions $f(z)$ and $g(z)$ of a Weierstrass equation. They are thought of as complex codimension-1 objects and correspond to the two kinds of critical points of a dessin d'enfant of Grothendieck. The $\mathbb{P}^{1}$ base is divided into several cell regions bounded by some domain walls extending from these planes and D-branes, on which the imaginary part of the $J$ function vanishes. This amounts to drawing a dessin with a canonical triangulation. We show that the dessin provides a new way of keeping track of mutual nonlocalness among 7-branes without employing unphysical branch cuts or their base point. With the dessin, we can see that weak- and strongcoupling regions coexist and are located across an $S$ wall from each other. We also present a simple method for computing a monodromy matrix for an arbitrary path by tracing the walls it goes through.
\end{abstract}

DOI: $10.1103 /$ PhysRevD.100.126025

\section{INTRODUCTION}

The importance of $\mathrm{F}$ theory [1-3] in modern particle physics model building cannot be emphasized too much. The $S U(5)$ grand unified theory (GUT), which can naturally explain the apparently complicated assignment of hypercharges to quarks and leptons, is readily achieved in $\mathrm{F}$ theory. Another virtue of $\mathrm{F}$ theory is that it can yield matter in the spinor representation of $S O(10)$, into which all the quarks and leptons of a single generation are successfully incorporated and which cannot be achieved in pure D-brane models. These features are shared by $E_{8} \times E_{8}$ heterotic models, but F-theory models have an advantage in that they may evade the issue of the relation between the GUT and Planck scales in heterotic string theory first addressed in Ref. [4]. Also, the Yukawa couplings perturbatively forbidden in D-brane models $[5,6]$ can be successfully generated in F theory.

Almost ten years after the first development in F theory, there was much progress in the studies of local models of $F$ theory (see Refs. [7-16] for an incomplete list.). In this class

\footnotetext{
*fshin@ post.kek.jp

†naotok@post.kek.jp

\#mizoguch@ post.kek.jp

\$tashiro@post.kek.jp
}

Published by the American Physical Society under the terms of the Creative Commons Attribution 4.0 International license. Further distribution of this work must maintain attribution to the author(s) and the published article's title, journal citation, and DOI. Funded by SCOAP ${ }^{3}$. of theories, one basically considers a supersymmetric gauge theory ${ }^{1}$ on a stack of 7-branes in F theory, the coalescence of which is supposed to give rise to a gauge symmetry depending on the fiber type in the Kodaira classification. In particular, if the fiber type is either $I V^{*}, I I I^{*}$, or $I I^{*}$, the gauge symmetry will be $E_{6}, E_{7}$, or $E_{8}$, respectively, and then the brane is called an exceptional brane [8]. ${ }^{2}$

The fiber type of such a codimension-1 singularity can be labeled by the (conjugacy class of the) $\operatorname{SL}(2, \mathbb{Z})$ monodromy around the fiber. It was shown that all the types of Kodaira fibers can be represented by some product of monodromies of a basic set of 7-branes: $\mathbf{A}=\mathrm{D}$-brane, $\mathbf{B}=(1,1)$-brane, and $\mathbf{C}=(1,-1)$-brane [51-53], as shown in Table I in the Appendix. ${ }^{3}$ The relation between the resolution of the singularity and the gauge symmetry on a coalescence of 7-branes has been clearly explained by using string junctions. String junctions are also useful to describe chiral matter [55], non-simply-laced Lie algebras [56], the Mordell-Weil lattice of a rational elliptic surface [57], and deformations of algebraic varieties [58,59].

\footnotetext{
${ }^{1}$ More precisely, the compact part of the theory is "twisted" so that the Casimirs of the gauge fields correctly transform as sections of Looijenga's weighted projective space bundle [17].

${ }^{2}$ More recently, after the LHC run in particular, global F-theory models have been attracting much interest. For recent works on global F-theory models, see, e.g., Refs. [18-50].

${ }^{3}$ In this paper, we identify these 7-branes as the monodromy matrices $M_{p, q}$ defined in Ref. [51] with the sign of $q$ reversed (as we have adopted Schwarz's convention for the tension [54]), which are the inverse of $K_{[p, q]}$ in Refs. [52,53]; this is consistent, as the orderings of the branes and $K_{[p, q]}$ 's are the reverse of each other.
} 
From Table I, can see that the singular fibers of the exceptional type consist of a $\mathbf{B}$-brane and two $\mathbf{C}$-branes in addition to the ordinary $\mathrm{D}(=\mathbf{A})$-branes. Thus, in this algebraic approach, the exceptional branes are seen to emerge due to the coalescence of these $\mathbf{B}$ - and $\mathbf{C}$-branes, which are distinct from D-branes. From a geometrical point of view, however, these branes are just the zero loci of the discriminant of a Weierstrass equation, and there are no a priori differences from each other; they all are locally D-branes.

In this paper, we consider the special roles of the zero loci of the Weierstrass invariants $g_{2}(\tau(z)), g_{3}(\tau(z))$ in $\mathrm{F}$ theory on an elliptic fibration over $\mathbb{P}^{1}$, or a further fibration thereof. They are defined as the zero loci of the coefficient functions $f(z)$ and $g(z)$ of a Weierstrass equation. They are thought of as complex codimension-1 objects, and we call them "elliptic point planes."

In fact, mathematically, our construction amounts to drawing a "dessin d'enfant" of Grothendieck on the $\mathbb{P}^{1}$ base with a canonical triangulation. ${ }^{4}$ We show that this drawing provides a new way of keeping track of mutual nonlocalness among 7-branes in place of the conventional A BC 7-brane description. In our approach, all the discriminant loci are treated democratically, and with this dessin, we can see that weak- and strong-coupling regions coexist and are located across an $S$ wall from each other. We also present a simple method for computing a monodromy matrix for an arbitrary path by tracing the walls it goes through. The method for studying monodromies by tracing the contours on the $J(\tau)$ plane was developed long time ago by Tani [55].

This paper is organized as follows. In Sec. II, we introduce the basic setup of this paper, including the motivations and definitions of the elliptic point planes, the domain walls extended from them, and the cell region decomposition of the $\mathbb{P}^{1}$ base of the elliptic fibration. The various definitions of the new notions and objects are summarized as a miniglossary at the end of this section. In Sec. III, we briefly explain what is a dessin d'enfant and the relation to our present construction. In Sec. IV, we discuss the basic properties of the two kinds of elliptic point planes, the $f$ plane and the $g$ plane. In Sec. V, we present a new method for computing the monodromy by drawing the dessin. In the final section, we conclude with a summary of our findings. Appendix contains a table of fiber types of the Kodaira classification. The plots presented in this paper have been generated with the aid of Mathematica.

\section{WHAT IS AN ELLIPTIC POINT PLANE?}

Consider a Weierstrass equation

$$
y^{2}=x^{3}+f x+g,
$$

\footnotetext{
${ }^{4}$ We thank the anonymous referee of Physical Review D for informing us of this fact.
}

where $y, x, f$, and $g$ are sections of an $\mathcal{O}(3)$, an $\mathcal{O}(2)$, an $\mathcal{O}(4)$, and an $\mathcal{O}(6)$ bundle over the base $\mathbb{P}^{1}$. This is a rational elliptic surface, which we regard as one of the two rational elliptic surfaces arising in the stable degeneration limit of a K3 surface. It may also be thought of as the total space of a Seiberg-Witten curve (with the " $u$ " plane being the base) of an $\mathcal{N}=2 S U(2)$ gauge theory or an E-string theory. In an affine patch of $\mathbb{P}^{1}$ with the coordinate $z$, the coefficient functions $f(z)$ and $g(z)$ are a fourth- and a sixth-order polynomial in $z^{5}$

As is well known, the modulus $\tau$ of the elliptic fiber of (1) is given by the implicit function

$$
J(\tau)=\frac{4 f^{3}}{4 f^{3}+27 g^{2}},
$$

where $J$ is the elliptic modular function. The denominator of the right-hand side

$$
\Delta \equiv 4 f^{3}+27 g^{2}
$$

is called the discriminant. Near its zero locus $z=z_{i}, \operatorname{Im} \tau$ goes to $\infty$ (if one has chosen the "standard" fundamental region) for generic (that is, nonzero) $f$ and $g$. Examining the behavior of $J(\tau)$ around $\infty$, we find

$$
\tau(z)=\frac{1}{2 \pi i} \log \left(z-z_{i}\right)\left(\text { const }+O\left(z-z_{i}\right)\right),
$$

which implies the existence of a D7-brane at each discriminant locus. ${ }^{6}$

On the other hand, since a locus of $f(z)=0$ or $g(z)=0$ alone does not mean $\Delta=0$, it is not a D-brane. However, if the loci of $f(z)=0$ and $g(z)=0$ are present together with a D-brane, they play a significant role in generating a $(p, q)$-7-brane by acting $S L(2, \mathbb{Z})$ conjugate transformations on a D-brane or as components of an orientifold plane, as we show below. In this paper, we will collectively call the loci of $f(z)=0$ and $g(z)=0$ elliptic point planes. ${ }^{7}$

\footnotetext{
${ }^{5}$ Although we introduce and define various notions in this simple setup, most of them can be generalized to a lowerdimensional F-theory compactification on a higher-dimensional elliptic Calabi-Yau, the base $\mathcal{W}$ of which is a $\mathbb{P}^{1}$ fibration over some base manifold $\mathcal{B}$, by simply taking $y, x, f$, and $g$ to be sections of $K_{\mathcal{W}}^{-3}, K_{\mathcal{W}}^{-2}, K_{\mathcal{W}}^{-4}$, and $K_{\mathcal{W}}^{-6}$, respectively, where $K_{\mathcal{W}}$ is the canonical class of $\mathcal{W}$. Equation (1) then describes a K3 fibered Calabi-Yau over $\mathcal{B}$. A configuration of the elliptic point planes, D-branes, and various walls are then a "snapshot" of a $\mathbb{P}^{1}$ fiber over some point on $\mathcal{B}$ with fixed coordinates.

${ }^{6}$ Thus, henceforth in this paper, we refer to a locus of the discriminant as (a locus of) a D-brane. As we will see, however, the monodromy around it is not always $T$ (13) for a general choice of the reference point, due to the presence of the elliptic point planes.

${ }^{7}$ In the standard fundamental region of the modular group of a 2-torus, there are two elliptic points $\tau=e^{\frac{2 \pi i}{3}}$ and $i$. They are fixed points of actions of some elliptic elements of $S L(2, \mathbb{Z})$, hence the name.
} 
Elliptic point planes consist of two types, the loci of $f(z)=0$ and $g(z)=0$, which have different properties. In this paper, we call the locus of $f(z)=0$ an $f=0$ "locus plane", or an " $f$ plane" for short, and that of $g(z)=0$ a $g=0$ locus plane, or a " $g$ plane" for short. ${ }^{8}$

At the location of an $f$ plane, the value of the $J$ function is

$$
J(\tau)=\frac{4 f^{3}}{4 f^{3}+27 g^{2}}=0,
$$

which corresponds to $\tau=e^{\frac{2 \pi i}{3}}$. On the other hand, at the position of a $g$ plane,

$$
J(\tau)=\frac{4 f^{3}}{4 f^{3}+27 g^{2}}=1,
$$

so this implies $\tau=i$. In their neighborhoods, $J(\tau)$ is expanded as

$$
\begin{aligned}
& J(\tau)=\frac{1}{3 !} J^{\prime \prime \prime}\left(e^{\frac{2 \pi i}{3}}\right)\left(\tau-e^{\frac{2 \pi i}{3}}\right)^{3}+O\left(\left(\tau-e^{\frac{2 \pi i}{3}}\right)^{4}\right), \\
& J(\tau)=1-\frac{12 K\left(\frac{1}{\sqrt{2}}\right)^{4}}{\pi^{2}}(\tau-i)^{2}+O\left((\tau-i)^{3}\right),
\end{aligned}
$$

where $K(k)$ is the complete elliptic integral of the first kind,

$$
K(k)=\int_{0}^{\frac{\pi}{2}} \frac{d \theta}{\sqrt{1-k^{2} \sin ^{2} \theta}} .
$$

Thus, $\tau=e^{\frac{2 \pi i}{3}}$ is a triple zero of $J(\tau)$, and $\tau=i$ is a double zero of $J(\tau)-1$.

Suppose that $z=0$ is a locus of $f=0$. Since

$$
J(\tau(z))=\frac{4 f(z)^{3}}{4 f(z)^{3}+27 g(z)^{2}},
$$

$J(\tau(z))$ is $O\left(z^{3}\right)$ at $z=0$. So, Eq. (7) shows that $\tau-e^{\frac{2 \pi i}{3}}$ is $O(z)$ there, implying that the monodromy is trivial around the locus of $f$. Similarly, if $z=0$ is a locus of $g=0$, $J(\tau(z))-1$ is now $O\left(z^{2}\right)$. Comparing this with (8), we see that $\tau(z)-i$ is also $O(z)$, and hence there is no monodromy around the locus of $g=0$, either.

However, this is not the end of the story. Figure 2 shows the various choices of fundamental regions of the modulus $\tau$ and the corresponding complex plane as its image mapped by the $J$ function. From this, we can see that if one goes around $\tau=e^{\frac{2 \pi i}{3}}$ once on the upper half-plane, one

\footnotetext{
${ }^{8}$ Despite the name "plane," an elliptic point plane is no more a rigid object but a smooth submanifold when the elliptic fibration over $\mathbb{P}^{1}$ is further fibered over another manifold, just like a D-brane.
}

goes through three different fundamental regions to get back to the original position. Likewise, if one goes around $\tau=i$, one undergoes two different fundamental regions. Thus, an $f$ plane is a complex codimension-1 submanifold at which three different regions on the $z$ plane corresponding to different fundamental regions meet, while a $g$ plane is similarly the place where two different regions meet. The regions on the $z$ plane corresponding to different fundamental regions are bounded by real codimension- 1 domain walls, which consist of the zero loci of the imaginary part of the $J$ function.

Furthermore, each region on the $z$ plane corresponding to a definite fundamental region is divided by a domain wall,

$$
\{\tau \mid \operatorname{Im} J(\tau)=0, \operatorname{Re} J(\tau)>1\}
$$

(a dashed green line), into two regions $\operatorname{Im} J(\tau)>0$ and $\operatorname{Im} J(\tau)<0$.

On the other hand, a D-brane resides at a discriminant locus $\Delta=0$, from which two domain walls $\{\tau \mid \operatorname{Im} J(\tau)=0$, $\operatorname{Re} J(\tau)<0\}$ (a green line) and $\{\tau \mid \operatorname{Im} J(\tau)=0, \operatorname{Re} J(\tau)>1\}$ (a dashed green line) extend out into the bulk $z$ space $\left(\mathbb{P}^{1}\right)$ (Fig. 1).

Since the value of $J$ is $\infty$ at a discriminant locus for generic (i.e., nonzero) values of $f$ and $g$, D-branes can never, by definition, touch nor pass through (a non-endpoint of) the domain walls because $\operatorname{Im} J(\tau)$ must vanish at the domain walls.

In this way, the $z$ space $\left(=\mathbb{P}^{1}\right)$ is divided into several cell regions, which correspond to different fundamental regions in the preimage of the $J$ function, by the domain walls extended from the elliptic point planes ( $f$ planes and $g$ planes) and D-branes (Fig. 1). In particular, $f$ planes and $g$ planes extend the domain walls

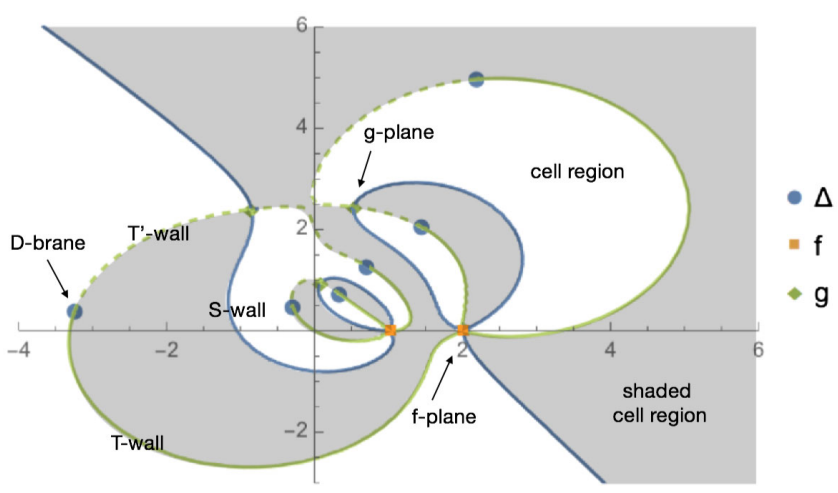

FIG. 1. An example configuration of D-branes, elliptic point planes, and the cell regions bounded by the domain walls extended from them. D-branes are located at the loci of $\Delta=0$, while elliptic point planes are at the loci of $f=0$ and $g=0$. In this example, we can see two $f$ planes at $z=1,2$, three $g$ planes, and six D-branes. [This figure is depicted for the Weierstrass equation (1) for $f$ and $g$ (43) with $\epsilon=0.9$ ]. 


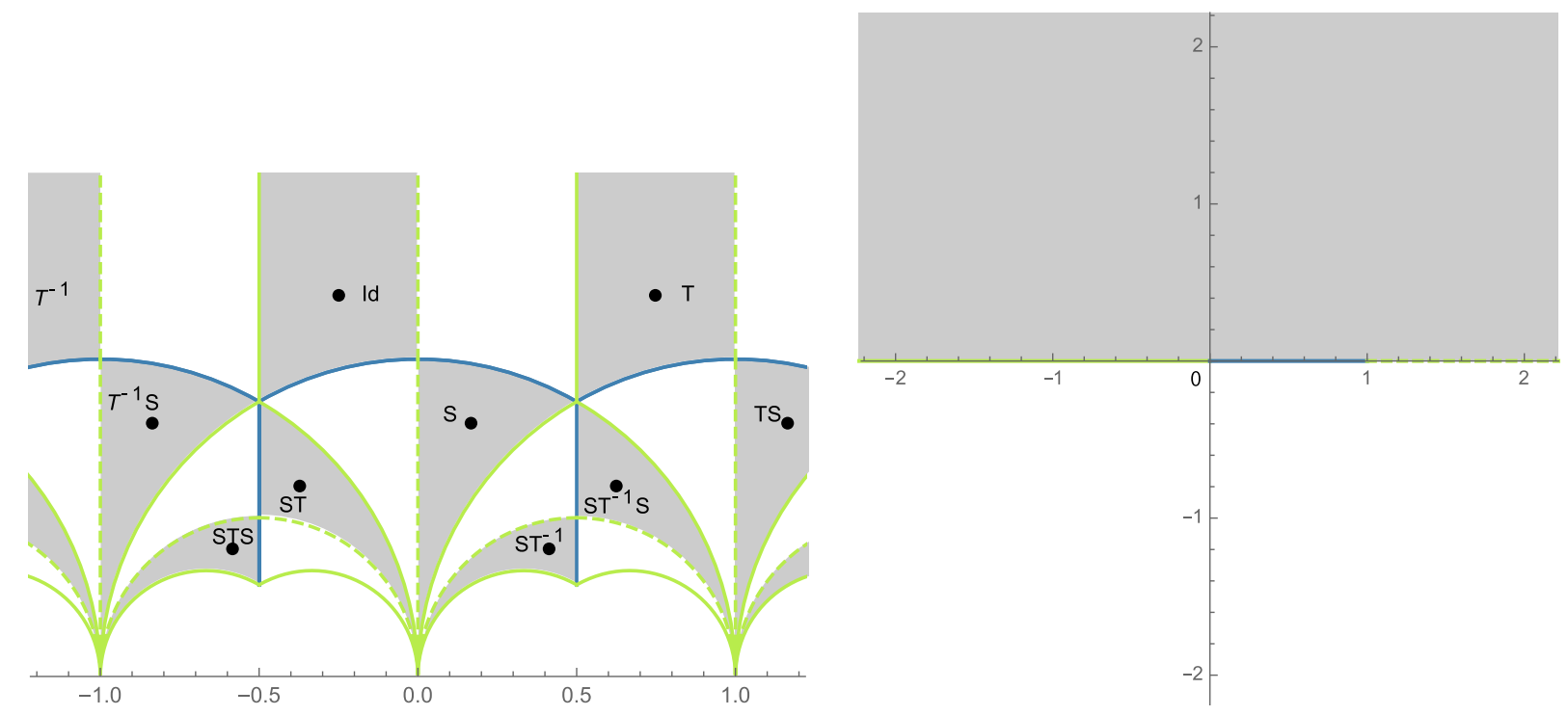

FIG. 2. Left: The upper half-plane and various fundamental regions. The shaded regions are the regions in which the imaginary part of the image of the $J$ function $\operatorname{Im} J(\tau)$ is positive. The symbol in each fundamental region (such as Id, $T, S, \ldots$ ) is the group element of $S L(2, \mathbb{Z})$ that maps the standard fundamental region to the fundamental region specified by the symbol. Right: The images of the $J$ function (the whole complex plane). The green, blue, and dashed green lines correspond to the respective boundary components of any one half of (the closure of) the fundamental regions.

$$
\{\tau \mid \operatorname{Im} J(\tau)=0, \quad 0<\operatorname{Re} J(\tau)<1\}
$$

(blue lines), and crossing through this wall implies that the type IIB coupling locally gets $S$ dualized (if starting from the standard choice of the fundamental region) (Fig. 2). Then, there is a difference in monodromies between when one goes around a D-brane within a single cell region bounded by some domain walls and when one first crosses through a domain wall, moves around a D-brane, and then crosses back through the wall again to the original position; they are different by an $S L(2, \mathbb{Z})$ conjugation. This is what is happening in what has been called a "B-brane" or a "C-brane" in the discussions of string junctions. That is, while the monodromy matrix is necessarily

$$
T=\left(\begin{array}{ll}
1 & 1 \\
0 & 1
\end{array}\right)
$$

as long as the reference point is chosen to be in the standard fundamental region, a nontrivial (non-D-brane) $(p, q)$ brane arises if the monodromy is measured by going back and forth between regions corresponding to different fundamental regions in the preimage upper half-plane.

We would like to emphasize here that such a local $S$ transformation never takes place without these elliptic point planes ( $f$ planes and $g$ planes). If there are no elliptic point planes but there are only D-branes, the domain walls extended from them are only the ones

$$
\{\tau \mid \operatorname{Im} J(\tau)=0, \operatorname{Re} J(\tau)<0\}
$$

(green lines) and

$$
\{\tau \mid \operatorname{Im} J(\tau)=0, \operatorname{Re} J(\tau)>1\}
$$

(dashed green lines). So, crossing through these walls only leads to a $T$ transformation which commutes with the original monodromies of D-branes.

In the discussion below, we refer to the domain wall (14) (a green lines) as $T$ wall and the one in (15) (a dashed green line) as $T^{\prime}$ wall, whereas we call the type of domain wall in (12) (a blue line) $S$ wall.

To conclude this section, we summarize the definitions of the new objects and notions introduced in this section as a miniglossary:

(i) The $f$ plane is a (complex) codimension- 1 object corresponding to a zero locus of $f(z)$ in the Weierstrass form on the $z$ plane, represented by a small square in the figures.

(ii) The $g$ plane is a (complex) codimension-1 object corresponding to a zero locus of $g(z)$ in the Weierstrass form on the $z$ plan, represented by a small $45^{\circ}$-rotated square in the figures.

(iii) The elliptic point plane is the collective name for $f$ planes and $g$ planes.

(iv) The $T$ wall is a (real) codimension- 1 object (domain wall) corresponding to a zero locus of $\operatorname{Im} J$ with $\operatorname{Re} J<0$, extending from a D-brane and a $f$ plane, represented by a green line.

(v) The $T^{\prime}$ wall is a (real) codimension-1 object (domain wall) corresponding to a zero locus of $\operatorname{Im} J$ with 

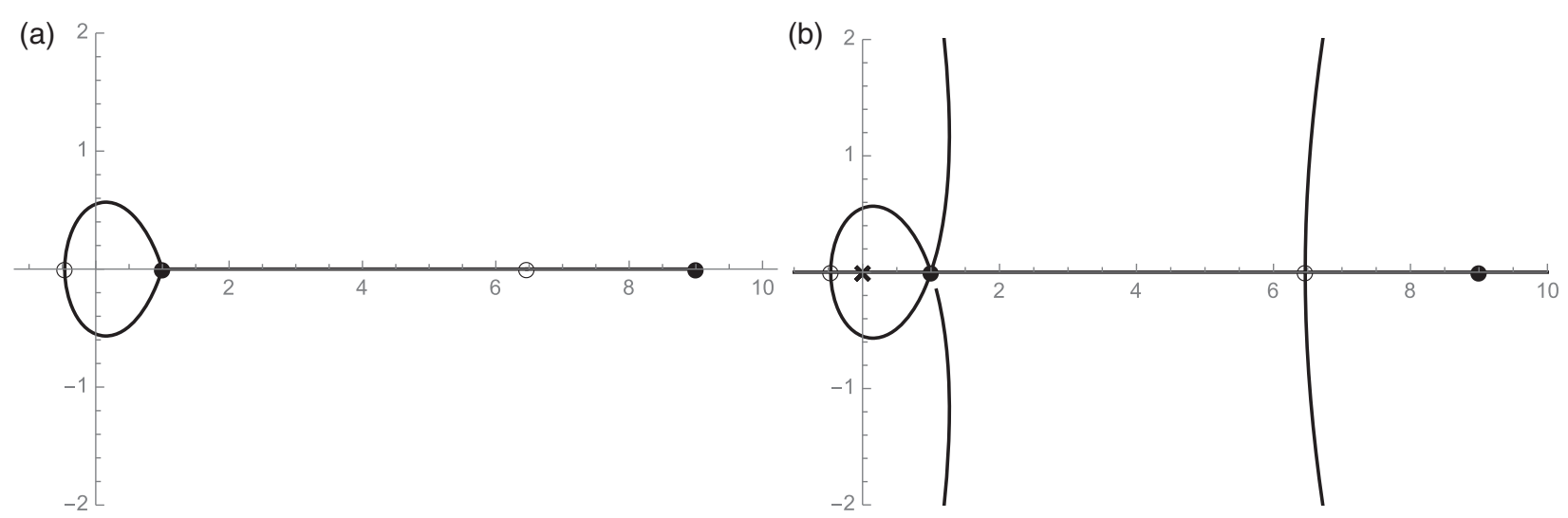

FIG. 3. (a) The dessin for (16). (b) The triangulated dessin. $\times$ represents an $\infty$ point. The extra lines have been drawn at the preimages of the segment $[-\infty, 0]$ and $[1, \infty]$. The other $\infty$ point is not shown in this figure, as it is infinitely far away.

$\operatorname{Re} J>1$, extending from a D-brane and a $g$ plane, represented by a dashed green line.

(vi) The $S$ wall is a (real) codimension-1 object (domain wall) corresponding to a zero locus of $\operatorname{Im} J$ with $0<\operatorname{Re} J<1$, extending from a $f$ plane and a $g$ plane, represented by a blue line.

(vii) The cell region is a closed region on the $z$ plane $\left(\mathbb{P}^{1}\right.$ base of the elliptic fibration) bounded by the $T, T^{\prime}$, and $S$ walls. Each cell region corresponds to either half of the (closure of the $)^{9}$ fundamental region with $\operatorname{Im} J>0$ or $\operatorname{Im} J<0$ of the fiber modulus.

(viii) The shaded cell region is the cell region corresponding to the (closure of the) half fundamental region with $\operatorname{Im} J>0$ (Fig. 1).

\section{RELATION TO DESSIN D'ENFANT OF GROTHENDIECK}

In fact, the construction in the previous section is nothing but drawing a dessin d'enfant of Grothendieck [60], known in mathematics, on the $\mathbb{P}^{1}$ base with a canonical triangulation. ${ }^{10} \mathrm{~A}$ dessin d'enfant, meaning a drawing of a child, is a graph consisting of some black points, white points, and lines connecting these points, drawn according to a special rule. To demonstrate the rule, let us consider, for example, a function [61],

$$
F(x)=-\frac{(x-1)^{3}(x-9)}{64 x}=1-\frac{\left(x^{2}-6 x-3\right)^{2}}{64 x},
$$

where $x \in \mathbb{P}^{1} . F$ is a map from $\mathbb{P}^{1}$ to $\mathbb{P}^{1}$. At almost everywhere on $\mathbb{P}^{1}, F$ is a homeomorphism, sending a small disk to another in a one-to-one way. However, $F$ maps a small disk centered at $x=1$ to one centered at $F=0$ in a

\footnotetext{
${ }^{9}$ Below, we abuse terminology and refer to a "fundamental region" as one modulo points on its boundary.

${ }^{10}$ The contents of this section are triggered by a suggestion made by the anonymous referee of Physical Review D.
}

three-to-one way. Similarly, $F$ is a two-to-one map from a small disk centered at $x=3 \pm 2 \sqrt{3}$ to one centered at $F=1$. The points $x=1,3 \pm 2 \sqrt{3}$ are said critical points, and the corresponding values of $F$ are said critical values. If the map from the neighborhood around a critical point to another around the corresponding critical value is $k$ to one, we say that the ramification index of the critical point is $k$.

Now, the rule to draw the dessin associated with (16) is as follows. Place a black point at every preimage of 0 and a white point at every preimage of 1 . Next, draw lines at preimages of the line segment $[0,1]$. The result is shown in Fig. 3(a).

Equation (16) induces a branched covering over $\mathbb{P}^{1}$. Treating this graph as a combinatorial object, one can reproduce the information of the branched covering as follows. One first adds a point $\infty$ to each region of the dessin. One then connects each $\infty$ with lines to the black or white points as many times as they appear on the boundary of the region. This yields a triangulation of the dessin. Assigning either the upper or the lower half-plane to each triangle depending on the ordering of $0,1, \infty$ and gluing these half-planes together, one obtains a branched covering equivalent to the original one [61].

In the present case, Eq. (10) defines a Belyi function, a holomorphic function of which the critical values are only 0,1 , and $\infty$ and nothing else. The black and white points in the dessin shown in Fig. 3(a) correspond to the $f$ planes and $g$ planes. The points $\infty$ added in the triangulation of the dessin are D-branes. The lines shown in Fig. 3(a) are the $S$ walls, while the lines connecting the $\infty$ points and the black or white points drawn in the triangulation are the $T$ and $T^{\prime}$ walls.

What is special about (10) is that it induces a local homeomorphism between the $\mathbb{P}^{1}$ base and the upper halfplane. Indeed, as we saw in the previous section, the correspondence is one-to-one everywhere, even in the vicinity of the elliptic orbits $\tau=e^{\frac{2 \pi i}{3}}$ and $i$. This is so because the $J=0(f=0)$ points are always critical points with ramification index 3 , and the $J=1(g=0)$ points are 
always with ramification index 2 . In this paper, we treat the dessin not as just a combinatorial graph but draw the $\infty$ points and the triangulating lines (the $T$ and $T^{\prime}$ walls) also as preimages of the $J$ function, as shown in Fig. 3(b). The special feature of (10) then allows us to use the (triangulated) dessin as a convenient tool to compute monodromies, as we see below.

\section{BASIC PROPERTIES OF ELLIPTIC POINT PLANES}

\section{A. Basic properties of $\boldsymbol{f}$ planes}

As we defined in the previous sections, there are two kinds of elliptic point planes: $f$ planes and $g$ planes. In this section, we describe the basic properties of $f$ planes.

As the name indicates, $f$ planes are the loci where the function $f$ vanishes. As we saw in the previous section, these are the places where the $J$ function vanishes and $\tau$ becomes $e^{\frac{2 \pi i}{3}}$ [or its $S L(2, \mathbb{Z})$ equivalents].

As we saw in the previous section, the expansion of $J(\tau)$ near $\tau=e^{\frac{2 \pi i}{3}}$ is given by (7). If there is an $f$ plane at $z=0$, $f=0$ there, yielding

$$
\begin{gathered}
f(z)=f_{41} z+f_{42} z^{2}+\cdots, \\
g(z)=g_{60}+g_{61} z+g_{62} z^{2}+\cdots,
\end{gathered}
$$

where $f_{4 i}, g_{6 j}$ are constants with indices running over $i=$ $1, \ldots, 8$ and $j=1, \ldots, 12$ for a $\mathrm{K} 3$ surface and $i=1, \ldots, 4$ and $j=1, \ldots, 6$ for a rational elliptic surface. Since

$$
\frac{4 f^{3}}{4 f^{3}+27 g^{2}}=\frac{4 f_{41}^{3}}{27 g_{60}^{2}} z^{3}(1+O(z))
$$

$\tau(z)$ asymptotically approaches

$$
\tau(z)=e^{\frac{2 \pi i}{3}}+\frac{2 f_{41}}{\left(9 g_{60}^{2} J^{\prime \prime \prime}\left(e^{\frac{2 \pi i}{3}}\right)\right)^{\frac{1}{3}}} z
$$

as $z \rightarrow 0$. Therefore, $\tau$ is regular near $z=0$, and hence an $f$ plane does not carry D-brane charges.

Parametrize a small circle around $z=0$ by $z=\epsilon e^{i \theta}$ $(\epsilon>0)$; then, if one goes around along it once, so does $\tau$ go once around $e^{\frac{2 \pi i}{3}}$ along a small circle with a radius $\epsilon\left|\frac{2 f_{41}}{\left(9 g_{60}^{2} J^{\prime \prime \prime}\left(e^{\frac{2 \pi i}{3}}\right)\right)^{\frac{1}{3}}}\right|$. Thus, although the monodromy around an $f$ plane is trivial, one passes through the boundary of the half-fundamental region six times on the upper halfplane as one goes once around an $f$ plane. Since the neighborhoods of $z=0$ and $\tau=e^{\frac{2 \pi i}{3}}$ are homeomorphic, the neighborhood of $z=0$ around an $f$ plane is also divided into six cell regions corresponding to different half-fundamental regions. The six domain walls separating these cell regions consist of three $S$ walls (blue) with $(0<\operatorname{Re} J(\tau)<1)$ and three $T$ walls (green) $(\operatorname{Re} J(\tau)<0)$, which are extended alternately from the $f$ plane, forming a locally $\mathbb{Z}_{3}$-symmetric configuration.

On the upper half-plane, if one starts from the standard fundamental region and passes through preimages (of the $J$ function) of a $T$ wall (green) and an $S$ wall (blue) to go to the $S L(2, Z)$ equivalent point, then the $S L(2, \mathbb{Z})$ transformation mapping the original point to the final point is $T^{-1} S$. Further, if one crosses through preimages of a $T$ wall (green) and an $S$ wall (blue) again, the transformation to the final $S L(2, Z)$ equivalent point is $\left(T^{-1} S\right)^{2}=-S T \sim S T$ [as $\operatorname{PSL}(2, \mathbb{Z})]$.

Since

$$
\left(T^{-1} S\right)^{3}=1
$$

$T^{-1} S$ generates a $\mathbb{Z}_{3}$ group, which is the isotropy group of the elliptic point $\tau=e^{\frac{2 \pi i}{3}}$. It is easy to show that this $T^{-1} S$ transformation acts on the neighborhood of this point as a $\frac{2 \pi i}{3}$ rotation. Therefore, the configuration of $\tau$ near an $f$ plane is locally invariant under the simultaneous actions of the spacial $\mathbb{Z}_{3}$ rotation and the $\mathbb{Z}_{3} S L(2, \mathbb{Z})$ transformation. The metric near an $f$ plane is locally $\mathbb{Z}_{3}$ invariant.

\section{B. Basic properties of $g$ planes}

Likewise, the expansion of $J(\tau)$ around $\tau=i$ is given by (8). Let a $g$ plane be at $z=0$ this time. $f(z)$ and $g(z)$ are expanded as

$$
\begin{gathered}
f(z)=f_{40}+f_{41} z+f_{42} z^{2}+\cdots, \\
g(z)=g_{61} z+g_{62} z^{2}+\cdots
\end{gathered}
$$

Since

$$
\frac{4 f^{3}}{4 f^{3}+27 g^{2}}=1-\frac{27 g_{61}^{2}}{4 f_{40}^{3}} z^{2}(1+O(z)),
$$

$\tau(z)$ approaches

$$
\tau(z)=i+\frac{3 i \pi^{\frac{1}{2}} g_{61}}{4 K\left(\frac{1}{\sqrt{2}}\right)^{2} f_{40}^{\frac{3}{2}}} z
$$

as $z \rightarrow 0$. Thus, $\tau$ is again regular near a $g$ plane; therefore, a $g$ plane does not have D-brane charges, either. The monodromy around a $g$ plane is also trivial, although if one goes around it, one will be passing through the $S$ walls (blue lines) and the $T^{\prime}$ walls (dashed green lines) alternately, twice for each.

Suppose that on the upper half-plane one starts from an arbitrarily given point near $\tau=i$ in the standard fundamental region with $\operatorname{Re} \tau<0$ and goes through the preimages of an $S$ wall and a $T^{\prime}$ wall to reach the $S L(2, \mathbb{Z})$ equivalent point. This move can be achieved by the $S L(2, \mathbb{Z}) S$ transformation. This $S$ transformation acts on 
the neighborhood of $\tau=i$ as a $\mathbb{Z}_{2}$ rotation. The metric near a $g$ plane is also $S L(2, \mathbb{Z})$ invariant. Thus, the vicinity of a $g$ plane is invariant under the $\mathbb{Z}_{2}$ rotation associated with the $S$ transformation.

\section{SIMPLE METHOD TO COMPUTE THE MONODROMY USING THE DESSIN}

Drawing the contours of the walls and the positions of the D-branes and elliptic point planes, we can have a figure of the complex plane divided into several cell regions such as Fig. 1, which we call a dessin. ${ }^{11}$ For a given Weierstrass equation, the dessin provides us with a very simple method to compute the monodromy matrices along an arbitrary path around branes on the complex plane (an affine patch of the $\mathbb{P}^{1}$ or the " $u$ plane" of a Seiberg-Witten curve).

\section{A. Method}

To illustrate the method, let us consider the SeibergWitten curve of $\mathcal{N}=2$ pure $\left(N_{f}=0\right) S U(2)$ supersymmetric gauge theory [62]. The equation is

$$
y^{2}=x^{3}-u x^{2}+x
$$

Taking $u$ as the coordinate $z$, we obtain a Weierstrass equation with

$$
f(u)=-\frac{1}{3} u^{2}+1, \quad g(u)=-\frac{2}{27} u^{3}+\frac{1}{3} u,
$$

the dessin of which is shown in the upper panel of Fig. 4. Let us compute the monodromy around each discriminant locus. Choosing a starting point near the left locus (shown as a cross), the left path crosses the walls as

$$
\rightarrow \mathbf{G} \rightarrow \mathbf{B} \rightarrow \mathbf{G} \rightarrow \mathbf{d G} \rightarrow,
$$

where $\mathbf{G}$ denotes the $T$ wall, B denotes the $S$ wall, and $\mathbf{d G}$ denotes the $T^{\prime}$ wall. ${ }^{12}$ The monodromy matrices for various patterns of crossings are

$$
\begin{aligned}
& \rightarrow \mathbf{d G} \rightarrow \mathbf{G} \rightarrow=T \\
& \rightarrow \mathbf{G} \rightarrow \mathbf{d G} \rightarrow=T^{-1} \\
& \rightarrow \mathbf{d G} \rightarrow \mathbf{B} \rightarrow=\rightarrow \mathbf{B} \rightarrow \mathbf{d G} \rightarrow=S \\
& \rightarrow \mathbf{B} \rightarrow \mathbf{G} \rightarrow=S T \\
& \rightarrow \mathbf{G} \rightarrow \mathbf{B} \rightarrow=T^{-1} S
\end{aligned}
$$

\footnotetext{
${ }^{11}$ This corresponds to a triangulated dessin in the sense of Grothendieck.

${ }^{12} \mathbf{G}, \mathbf{B}$, and $\mathbf{d G}$ are, respectively, the first letters of green, blue, and dashed green. We have avoided using $T, S$, or $T^{\prime}$ here, as the monodromy matrices for the crossing do not coincide with the names of the walls.
}

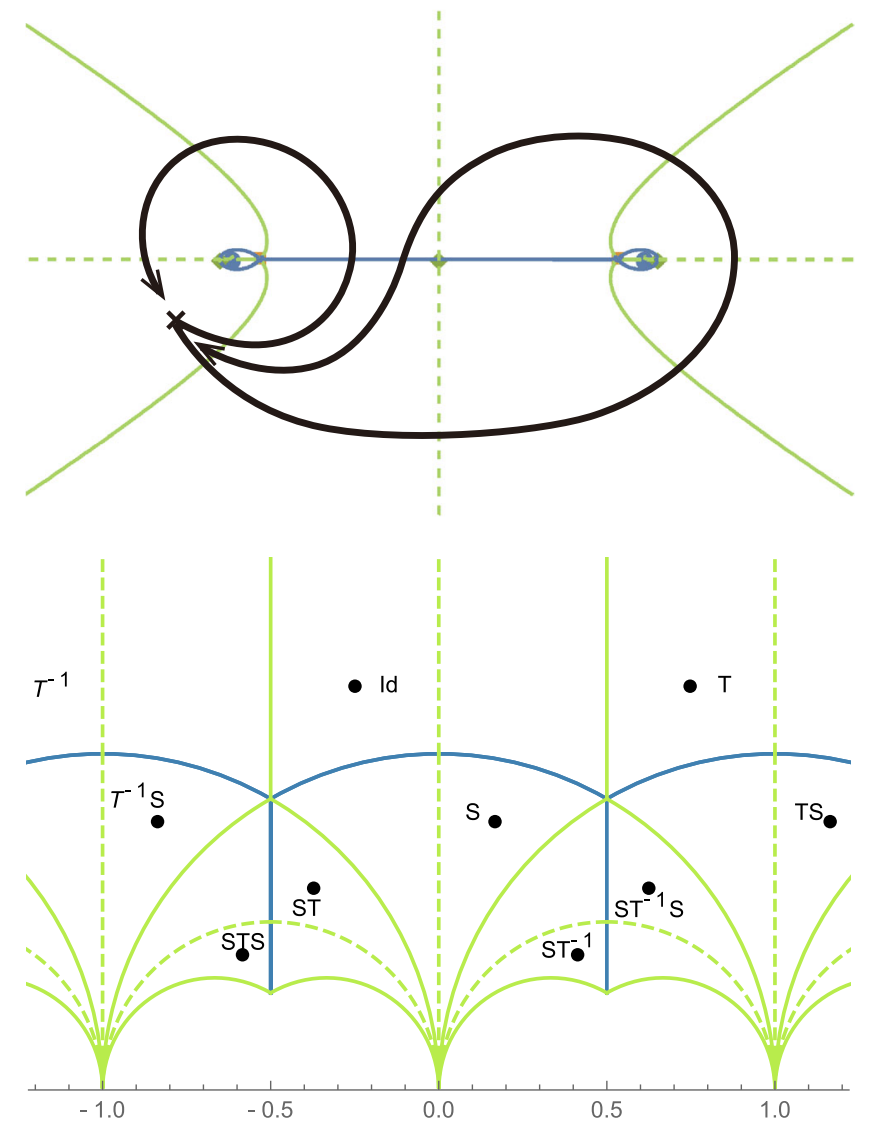

FIG. 4. Upper panel: The dessin of $N_{f}=0$ Seiberg-Witten curve $\left(f(u)=-\frac{1}{3} u^{2}+1, g(u)=-\frac{2}{27} u^{3}+\frac{1}{3} u\right)$. Lower panel: The crossed walls and the corresponding monodromies.

where the first wall of each row is the crossing from a shaded cell region $(\operatorname{Im} J>0)$ to an unshaded one $(\operatorname{Im} J<0)$ and the second is from an unshaded to a shaded one. ${ }^{13}$ The monodromy matrices are defined as

$$
T=\left(\begin{array}{ll}
1 & 1 \\
0 & 1
\end{array}\right), \quad S=\left(\begin{array}{cc}
0 & -1 \\
1 & 0
\end{array}\right)
$$

as usual, where we say that the monodromy matrix is $\left(\begin{array}{ll}a & b \\ c & d\end{array}\right)$ if the modulus $\tau$ is changed to

$$
\tau^{\prime}=M \circ \tau \equiv \frac{a \tau+b}{c \tau+d} .
$$

They are defined only in $\operatorname{PSL}(2, \mathbb{Z})$, i.e., up to a multiplication of -1 .

\footnotetext{
${ }^{13}$ Therefore, these rules only apply when one computes a monodromy for a path that starts from and ends in a shaded cell region $(\operatorname{Im} J>0)$. The rules for computing a monodromy for a path from an unshaded cell region $(\operatorname{Im} J<0)$ to another are similar but different: $\rightarrow \mathbf{d G} \rightarrow \mathbf{G} \rightarrow=T^{-1}, \rightarrow \mathbf{G} \rightarrow \mathbf{d G} \rightarrow=$ $T, \quad \rightarrow \mathbf{d G} \rightarrow \mathbf{B} \rightarrow=\rightarrow \mathbf{B} \rightarrow \mathbf{d G} \rightarrow=S, \quad \rightarrow \mathbf{B} \rightarrow \mathbf{G} \rightarrow=S T^{-1}$, $\rightarrow \mathbf{G} \rightarrow \mathbf{B} \rightarrow=T S$.
} 
By using the rule (29), we can immediately find the monodromy matrix for the path (28) as

$$
T^{-1} S \cdot T^{-1}=T^{-1} S T^{-1} \sim S T S,
$$

where $\sim$ denotes the equality in $\operatorname{PSL}(2, \mathbb{Z})$.

Similarly, the crossed walls for the right path are

$$
\rightarrow \mathrm{G} \rightarrow \mathrm{dG} \rightarrow \mathrm{G} \rightarrow \mathrm{dG} \rightarrow \mathrm{G} \rightarrow \mathrm{dG} \rightarrow \mathrm{B} \rightarrow \mathrm{G} \rightarrow \mathrm{.}
$$

Using rule (29) again, we find that the monodromy is

$$
T^{-1} \cdot T^{-1} \cdot T^{-1} \cdot S T=T^{-3} S T .
$$

A confusing but important point of the rule is that, in the first example, the monodromy matrix $T^{-1}$, which corresponds to the crossings $\rightarrow \mathbf{G} \rightarrow \mathbf{d G} \rightarrow$ taking place after the crossings $\rightarrow \mathbf{G} \rightarrow \mathbf{B} \rightarrow$ is multiplied to $T^{-1} S$ from the right. This is confusing because if $M=\left(\begin{array}{ll}a & b \\ c & d\end{array}\right), M^{\prime}=\left(\begin{array}{l}a^{\prime} \\ c^{\prime}\end{array} b^{\prime}\right)$ and $\tau^{\prime}=M \circ \tau, \tau^{\prime \prime}=M^{\prime} \circ \tau^{\prime}$ then the monodromy matrix $M^{\prime \prime}=\left(\begin{array}{l}a^{\prime \prime} \\ c^{\prime \prime} \\ d^{\prime \prime}\end{array}\right)$ representing $\tau \mapsto \tau^{\prime \prime}=M^{\prime \prime} \circ \tau$ is given by

$$
M^{\prime \prime}=M^{\prime} M,
$$

in which $M^{\prime}$ is multiplied from the left.

More generally, the following statement holds: let $\gamma$ be a path specified by the series of the walls

$$
\gamma: \rightarrow \mathbf{W}_{1} \rightarrow \mathbf{W}_{2} \rightarrow \cdots \rightarrow \mathbf{W}_{k} \rightarrow
$$

where $\mathbf{W}_{i}(i=1, \ldots, k)$ are one of $\mathbf{G}, \mathbf{B}$, or $\mathbf{d G}$, and let $M_{\gamma}$ denote the associated monodromy matrix of $\gamma . k$ is an even positive integer. (If it is odd, a shaded cell region is mapped to an unshaded cell region or vice versa, and the transformation cannot be an $S L(2, \mathbb{Z})$ transformation). Let $\gamma_{1}$ and $\gamma_{2}$ be paths specified by the series of the walls crossed by them,

$$
\begin{aligned}
& \gamma_{1}: \rightarrow \mathbf{W}_{1}^{(1)} \rightarrow \mathbf{W}_{2}^{(1)} \rightarrow \cdots \rightarrow \mathbf{W}_{k_{1}}^{(1)} \rightarrow \\
& \gamma_{2}: \rightarrow \mathbf{W}_{1}^{(2)} \rightarrow \mathbf{W}_{2}^{(2)} \rightarrow \cdots \rightarrow \mathbf{W}_{k_{2}}^{(2)} \rightarrow,
\end{aligned}
$$

and let $\gamma_{1} \mapsto \gamma_{2}$ be the jointed path

$$
\begin{aligned}
\gamma_{1} \rightarrow \gamma_{2}: & \rightarrow \mathbf{W}_{1}^{(1)} \rightarrow \cdots \rightarrow \mathbf{W}_{k_{1}}^{(1)} \rightarrow \mathbf{W}_{1}^{(2)} \rightarrow \cdots \\
& \rightarrow \mathbf{W}_{k_{2}}^{(2)} \rightarrow
\end{aligned}
$$

where we use the new symbol $\rightarrow>$ to denote the operation of jointing two paths. ${ }^{14}$ Then, we have Proposition 1.

\footnotetext{
${ }^{14}$ We will not use the usual symbol for addition, + , since this operation is noncommutative.
}

\section{Proposition 1.}

$$
M_{\gamma_{1} \gg \gamma_{2}}=M_{\gamma_{1}} M_{\gamma_{2}}
$$

Remark 1.-As we noted above, the monodromy matrix corresponding to a later crossing comes to the right, unlike (35) in which the matrix for the later transformation is multiplied from the left.

Proof.-By induction with respect to the total number of crossed walls, it is enough to show the statement for the cases in which $\gamma_{2}$ is any of the crossing patterns (29). Suppose that $\gamma_{1}$ starts from a cell region $C_{0}$ and ends in another $C_{1}$ and that $\gamma_{2}$ goes from the cell region $C_{1}$ to another $C_{2}$, where $\gamma_{2}$ is taken to be any of the crossing patterns (29), say, $\gamma_{2}=\rightarrow \mathbf{d G} \rightarrow \mathbf{G} \rightarrow$ and $M_{\gamma_{2}}=T$. Let $P_{\gamma_{i}}(i=1,2$, respectively) be the associated maps which send points in the cell region $C_{i-1}$ to those in the cell region $C_{i}$, such that the torus modulus over the point is $S L(2, \mathbb{Z})$ equivalent. We say two points on $\mathbb{P}^{1}$ are $S L(2, \mathbb{Z})$ equivalent if the torus fiber moduli over them are $S L(2, \mathbb{Z})$ equivalent. Using this terminology, we can say that $P_{\gamma_{i}}(i=1,2)$ are the maps which send the points in $C_{i-1}$ to their $S L(2, \mathbb{Z})$-equivalent points in $C_{i}$, respectively. Since $\tau(z)$ is holomorphic in $z$ and $J(\tau)$ is holomorphic in $\tau$, the domain of the map $P_{\gamma_{1}}$ is not necessarily restricted to only $C_{0}$ but can be extended to outside $C_{0}$ as far as it is in a small neighborhood of $z_{0}$.

Let $z_{0}$ be a point in $C_{0}$, and let $z_{1}=P_{\gamma_{1}}\left(z_{0}\right) \in C_{1}$, $z_{2}=P_{\gamma_{2}}\left(z_{1}\right) \in C_{2}$. If we denote $\tau_{i}(i=0,1,2)$ be the modulus of the torus fiber over $z_{i}(i=0,1,2)$, they satisfy

$$
J\left(\tau_{i}\right)=\frac{4 f\left(z_{i}\right)^{3}}{4 f\left(z_{i}\right)^{3}+27 g\left(z_{i}\right)^{2}},
$$

where $\tau_{1}$ and $\tau_{2}$ are the values analytically continued from $\tau_{0}$ along the paths $\gamma_{1}$ and then $\gamma_{2}$. Taking $\tau_{0}$ in the standard fundamental region, the transformation from $\tau_{0}$ to $\tau_{1}$ is given by $\tau_{1}=M_{\gamma_{1}} \circ \tau_{0}$, but consecutive transformation from $\tau_{1}$ to $\tau_{2}$ is not $M_{\gamma_{2}} \circ \tau_{1}$, as $\tau_{1}$ does not belong to the standard fundamental region in general. Rather, since $P_{\gamma_{1}}$ is locally an isomorphism between a neighborhood around $z_{0}$ and that around $z_{1}$, the final point $z_{2}$ can be written as the $P_{\gamma_{1}}$ image of $z_{1}^{\prime}$, where $z_{1}^{\prime}$ is the $S L(2, \mathbb{Z})$ equivalent point in the cell region reached along the path $\gamma_{2}$ first from $z_{0}$, if $z_{2}$ is close enough to $z_{1}$ (Fig. 5). If, on the other hand, $z_{2}$ is not close to $z_{1}$, we can continuously deform the complex structure of the elliptic fibration so that $z_{2}$ may come close to $z_{1}$. Since this is a continuous deformation, the monodromy transformation matrix does not change, as the entries of the matrix take discrete values. Thus, we may assume that $z_{2}$ is close to $z_{1}$.

Since $\tau_{0}$ is taken in the standard fundamental region, $\tau_{1}^{\prime}$, the modulus of the torus fiber over $z_{1}^{\prime}$, is given by

$$
\tau_{1}^{\prime}=M_{\gamma_{2}} \circ \tau_{0}
$$




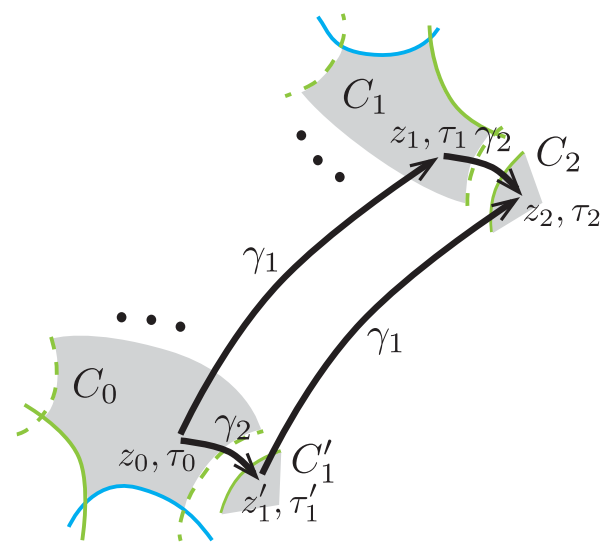

FIG. 5. Taking $\tau_{0}$ in the standard fundamental region, the transformation from $\tau_{0}$ to $\tau_{1}$ is given by $\tau_{1}=M_{\gamma_{1}} \circ \tau_{0}$, but consecutive transformation from $\tau_{1}$ to $\tau_{2}$ is not $M_{\gamma_{2}} \circ \tau_{1}$, as $\tau_{1}$ does not belong to the standard fundamental region in general. Rather, we have $\tau_{2}=M_{\gamma_{1}} \circ \tau_{1}^{\prime}$ with $\tau_{1}^{\prime}=M_{\gamma_{2}} \circ \tau_{0}$ as $P_{\gamma_{1}}$ induces an isomorphism.

Therefore, since $\tau_{2}=M_{\gamma_{1}} \circ \tau_{1}^{\prime}$, we find

$$
\tau_{2}=M_{\gamma_{1}} \circ M_{\gamma_{2}} \circ \tau_{0}=\left(M_{\gamma_{1}} M_{\gamma_{2}}\right) \circ \tau_{0}
$$

which is what the proposition claims.

In deriving (42), we did not use the fact that $\gamma_{2}$ was assumed to be a particular pattern among (29), but the relation (42) likewise holds for other patterns. This completes the proof of the proposition. ${ }^{15}$

\section{B. Example: Monodromies of $N_{f}=4 S U(2)$ Seiberg-Witten curves}

The proposition (39) together with the rule (29) provides us with a very convenient method to compute the monodromy for an arbitrary Weierstrass model along an arbitrary path.

Figure 6 is a dessin of the $N_{f}=4 S U(2)$ Seiberg-Witten curve with some mass parameters. The Weierstrass equation is (1) where

$$
\begin{aligned}
f= & (z-1)(z-2), \\
g= & \epsilon(z-i)(z-2 i)(z-3 i) \\
& +(1-\epsilon)\left(-\frac{5}{16} i \sqrt{\frac{3}{2} z^{3}}+\frac{17 i z^{2}}{4 \sqrt{6}}-i \sqrt{6} z+\frac{4}{3} i \sqrt{\frac{2}{3}}\right)
\end{aligned}
$$

with $\epsilon=3 \times 10^{-7}$. This choice of $g$ interpolates between the configuration in which all the $g$-locus planes are located

\footnotetext{
${ }^{15}$ In this proof, $\gamma_{2}$ is taken to be a path to the next adjacent cell region, whereas $\gamma_{1}$ is assumed to be some long path leading to a faraway cell region. If $\gamma_{1}$ is also a path to another next adjacent cell region, it can be explicitly checked that the proposition holds in this case as well.
}

on the imaginary axis at equal intervals $(\epsilon=1)$ and the one in which four of the six D-branes collide together at $z=0$ to form a $I_{4}$ singular fiber $(\epsilon=0)$, with the $f$ planes fixed at $z=1,2$. The figure is the configuration very close to the latter limit.

As is well known, the one-parameter (" $u$ ") family of tori describe the moduli space of the gauge theory and can be compactified into a rational elliptic surface by taking the variables and coefficient functions to be sections of appropriate line bundles, where the $u$ parameter becomes the affine coordinate $z$ of the base $\mathbb{P}^{1}$. Note, however, that the dessin can be drawn on this affine patch independently of the choices of the bundles; it only affects how many D-branes are at the infinity of $\mathbb{P}^{1}$.

This figure shows how the monodromies around the two D-branes on the right (located at $z \approx 1$ and $\approx 2$ ) change depending on the choice of the reference point. If it is taken far enough (as marked by a white star), the monodromies along the black contours read $M_{2,1}$ and $M_{0,1}$. This means that, as we show later, a $(2,1)$ and a $(0,1)$ string become light near the respective D-branes, showing that the locations of the D-branes are the $(2,1)$ dyon and the monopole point on the moduli space of the gauge theory, which is well known.

If the reference point is taken closer (as marked by a black star), then the monodromies along the dashed black contours are $M_{1,1}(=\mathbf{B})$ and $M_{1,-1}(=\mathbf{C})$, which agrees with the $\mathbf{A B C}$ brane description of the $I_{0}^{*}$ Kodaira singular fiber.

Finally, if the reference point is taken to be very close to the D-branes inside the cell regions surrounded by the $S$ walls, then the monodromies along the dotted contours are both $T$, showing that these branes look like ordinary D-branes if they are observed from very close to them.

\section{C. $(p, q)$-brane as an effective description}

Of course, it is well known that the monodromy changes depending the choice of the reference point. A monodromy matrix measured from some reference point gets $S L(2, \mathbb{Z})$ conjugated if it is measured from another point. What is new here that, by drawing a dessin, we can precisely see how and from where the monodromy matrix changes and gets conjugated as we vary the position of the reference point.

For instance, we can see from Fig. 6 that the monodromies around the two D-branes on the right are either $M_{2,1}, M_{0,1}$ or $M_{1,1}(=\mathbf{B}), M_{1,-1}(=\mathbf{C})$ for most choices of the reference point on the $z(\equiv u)$ plane, and they are recognized as ordinary $\left(M_{1,0}=\mathbf{A}\right)$ D-branes only when they are viewed from the points in the tiny regions surrounded by the $S$ walls. Thus, we see that the effective description of the two branes as $(1,1)(=\mathbf{B})$ - and $(1,-1)(=\mathbf{C})$-branes are good at the energy scale lower than the scale of the size of the small cell regions surrounded by the $S$ walls.

However, one can also set the mass parameters of the same gauge theory so that the dessin of the Seiberg-Witten 


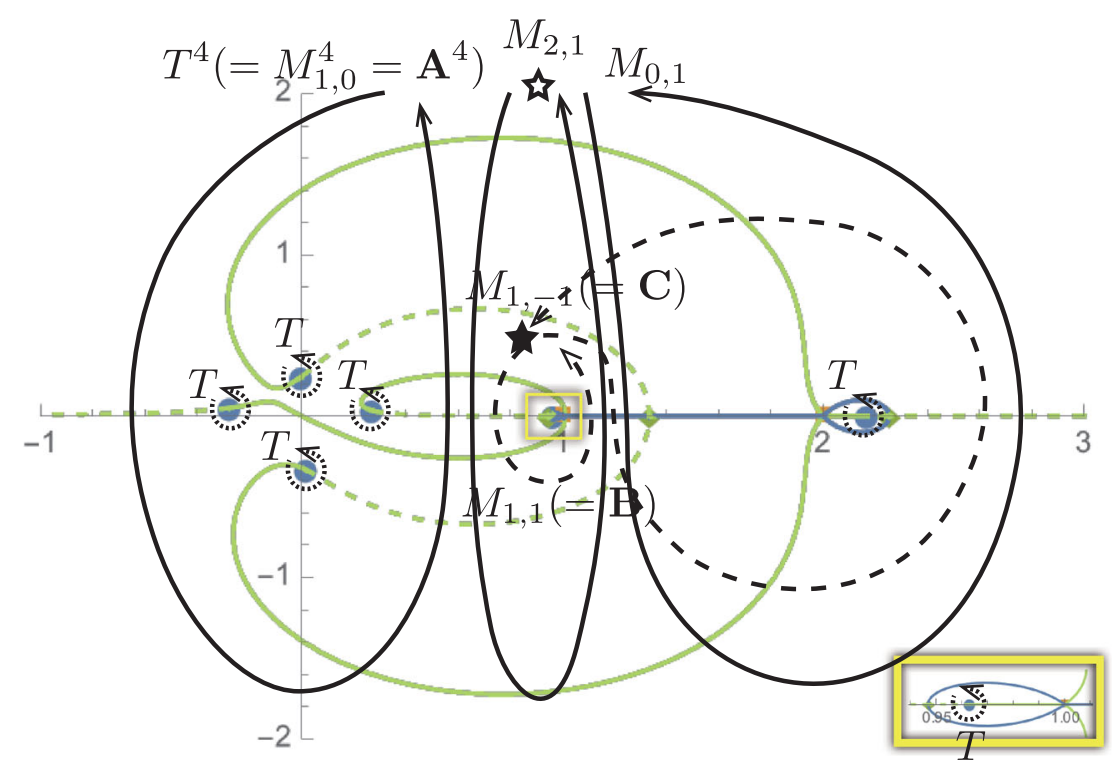

FIG. 6. Monodromies of $N_{f}=4 S U(2)$ Seiberg-Witten curve. It shows how the monodromies around the two D-branes on the right (located at $z \approx 1$ and $\approx 2$ ) change depending on the choice of the reference point. If it is taken far enough (as marked as a white star), the monodromies along the black contours read $M_{2,1}$ and $M_{0,1}$. If the reference point is taken closer (as marked by a black star), then the monodromies along the dashed black contours are $M_{1,1}(=\mathbf{B})$ and $M_{1,-1}(=\mathbf{C})$. If, on the other hand, the reference point is taken to be very close to the D-branes inside the cell regions surrounded by the $S$ walls, then the monodromies along the dotted contours are both $T$.

curve looks as shown in Fig. 1. In this case, the $S$ walls spread into wide areas of the $\mathbb{P}^{1}$. There is not much difference among the six D-branes, and there is no obvious reason to distinguish a particular two as $\mathbf{B}$ or $\mathbf{C}$ from the other four D-branes.

Remark 2.-We have seen that a cluster of a D-brane and two elliptic point planes, in which the former is surrounded by the $S$ walls extended from the latter, may be effectively identified as a $\mathbf{B}$ - or a $\mathbf{C}$-brane, if viewed from a distance of the size of the cluster. Thus, one might think that an "exact" $\left(p, q\right.$ )-brane (the monodromy of which is $M_{p, q}$ along an arbitrary small loop) can be obtained by taking the $f$ and $g$ planes on top of each other so that the size of the cell region the $S$ walls surround becomes zero. This is not the case, however, since if the $f$ and $g$ planes collide the order of the discriminant becomes 2, implying that another D-brane also automatically comes on top of the D-brane, $f$ plane, and $g$ plane. Since it contains two D-branes, it cannot be identified as a single $(p, q)$-brane in the $\mathbf{A B C}$-brane description.

\section{CONCLUSIONS}

The coexistence of D-branes and non-pure-D-7-branes is an essential feature of $\mathrm{F}$ theory, as it enables us to achieve exceptional group gauge symmetries or matter in spinor representations by allowing string junctions to appear as extra objects ending on more than two different types of 7-branes, in addition to the open strings which can only connect two ordinary D-branes. These 7-branes are conventionally described algebraically in terms of $\mathbf{A B C} 7$-branes. In this paper, noticing that all the discriminant loci are on equal footing and there is no $a$ priori reason to distinguish one from the others, we have considered new complex codimension-1 objects consisting of the zero loci of the coefficient functions $f$ and $g$ of the Weierstrass equation, which we referred to as an $f$ plane and a $g$ plane and collectively as elliptic point planes. They are two kinds of critical points of a dessin d'enfant known in mathematics.

Although they do not carry D-brane charges, they play an essential role in achieving an exceptional gauge symmetry and/or a spinor representation by altering the monodromies around the branes. More precisely, if there are some elliptic point planes, the $z$ plane is divided into several cell regions, each of which corresponds to a (half of a) fundamental region in the preimage of the $J$ function. A cell region is bounded by several domain walls extending from these elliptic point planes and D-branes, on which the imaginary part of the $J$ function vanishes. In particular, the elliptic point planes extend a special kind of domain walls, which we call $S$ walls, crossing through, which implies that the type IIB complex string coupling is $S$ dualized. Consequently, on the $z$ plane, a theory in the perturbative regime and its nonperturbative $S$ dual coexist simultaneously. The monodromy around several 7-branes is thus not just a product of monodromy around each 7-brane anymore, but they get $S L(2, \mathbb{Z})$ conjugated due to the difference of the corresponding fundamental regions to which the base points belong.

In this sense, one may say that the nonperturbative properties of $\mathrm{F}$ theory-the realizations of exceptional group symmetry, matter in spinor representations, etc.are the consequence of the coexisting "locally $S$-dualized regions" bounded by the $S$ walls extended from the elliptic 
point planes. In the orientifold limit [63], the D-branes and the elliptic point planes gather to form a $I_{0}^{*}$ singular fiber, so that the $S$ walls extended from the elliptic point planes are contracted with each other and confined, so the $S$ walls are not seen from even a short distance.

We hope this new way of presenting the nonlocalness among 7-branes will be useful for understanding of the structure of higher-codimension singularities with higherrank enhancement such as discussed in Refs. [2,3,11,64-67].

\section{ACKNOWLEDGMENTS}

We wish to thank the referee of Physical Review D for suggesting the improvement of the manuscript by considering the mathematical concept of dessin d'enfant. We also thank Y. Kimura and T. Tani for valuable discussions. The work of S.M. is supported by Grant-in-Aid for Scientific Research (C), Grant No. 16K05337, from The Ministry of Education, Culture, Sports, Science and Technology of Japan.

\section{APPENDIX: THE KODAIRA CLASSIFICATION}

TABLE I. The Kodaira classification. $\operatorname{ord}(f), \operatorname{ord}(g)$, and $\operatorname{ord}(\Delta)$ denote the orders of zeros of $f, g$, and the discriminant $\Delta$ of the Weierstrass equation.

\begin{tabular}{lcccccc}
\hline \hline $\begin{array}{l}\text { Fiber } \\
\text { type }\end{array}$ & $\begin{array}{c}\text { ord } \\
(f)\end{array}$ & $\begin{array}{c}\text { ord } \\
(g)\end{array}$ & $\begin{array}{c}\text { ord } \\
(\Delta)\end{array}$ & $\begin{array}{c}\text { Singularity } \\
\text { type }\end{array}$ & $\begin{array}{c}\text { 7-brane } \\
\text { configuration }\end{array}$ & $\begin{array}{c}\text { Brane } \\
\text { type }\end{array}$ \\
\hline$I_{n}$ & 0 & 0 & $n$ & $A_{n-1}$ & $\mathbf{A}^{n}$ & $A_{n-1}$ \\
$I I$ & $\geq 1$ & 1 & 2 & $A_{0}$ & $\mathbf{C A}$ & $H_{0}$ \\
$I I I$ & 1 & $\geq 2$ & 3 & $A_{1}$ & $\mathbf{C A}^{2}$ & $H_{1}$ \\
$I V$ & $\geq 2$ & 2 & 4 & $A_{2}$ & $\mathbf{C A}^{3}$ & $H_{2}$ \\
$I_{n}^{*}$ & $\geq 2$ & 3 & $6+n$ & $D_{n+4}$ & $\mathbf{A}^{n+4} \mathbf{B C}$ & $D_{n+4}$ \\
$I_{n}^{*}$ & 2 & $\geq 3$ & $6+n$ & $D_{n+4}$ & $\mathbf{A}^{n+4} \mathbf{B C}$ & $D_{n+4}$ \\
$I I^{*}$ & $\geq 4$ & 5 & 10 & $E_{8}$ & $\mathbf{A}^{7} \mathbf{B} \mathbf{C}^{2}$ & $E_{8}$ \\
$I I I^{*}$ & 3 & $\geq 5$ & 9 & $E_{7}$ & $\mathbf{A}^{6} \mathbf{B C}$ & $E_{7}$ \\
$I V^{*}$ & $\geq 3$ & 4 & 8 & $E_{6}$ & $\mathbf{A}^{5} \mathbf{B C}^{2}$ & $E_{6}$ \\
\hline \hline
\end{tabular}

[1] C. Vafa, Nucl. Phys. B469, 403 (1996).

[2] D. R. Morrison and C. Vafa, Nucl. Phys. B473, 74 (1996).

[3] D. R. Morrison and C. Vafa, Nucl. Phys. B476, 437 (1996).

[4] E. Witten, Nucl. Phys. B471, 135 (1996).

[5] R. Blumenhagen, B. Kors, D. Lust, and T. Ott, Nucl. Phys. B616, 3 (2001).

[6] R. Blumenhagen, M. Cvetic, D. Lust, R. Richter, and T. Weigand, Phys. Rev. Lett. 100, 061602 (2008).

[7] R. Donagi and M. Wijnholt, Adv. Theor. Math. Phys. 15, 1237 (2011).

[8] C. Beasley, J. J. Heckman, and C. Vafa, J. High Energy Phys. 01 (2009) 058.

[9] C. Beasley, J. J. Heckman, and C. Vafa, J. High Energy Phys. 01 (2009) 059.

[10] R. Donagi and M. Wijnholt, Adv. Theor. Math. Phys. 15, 1523 (2011).

[11] H. Hayashi, T. Kawano, R. Tatar, and T. Watari, Nucl. Phys. B823, 47 (2009).

[12] R. Donagi and M. Wijnholt, Commun. Math. Phys. 326, 287 (2014).

[13] J. J. Heckman, J. Marsano, N. Saulina, S. Schafer-Nameki, and C. Vafa, arXiv:0808.1286.

[14] J. Marsano, N. Saulina, and S. Schafer-Nameki, Phys. Rev. D 80, 046006 (2009).

[15] J. J. Heckman and C. Vafa, J. High Energy Phys. 09 (2009) 079.

[16] A. Font and L. E. Ibanez, J. High Energy Phys. 02 (2009) 016.

[17] R. Friedman, J. Morgan, and E. Witten, Commun. Math. Phys. 187, 679 (1997).

[18] H. Hayashi, R. Tatar, Y. Toda, T. Watari, and M. Yamazaki, Nucl. Phys. B806, 224 (2009).
[19] B. Andreas and G. Curio, J. Geom. Phys. 60, 1089 (2010).

[20] J. Marsano, N. Saulina, and S. Schafer-Nameki, J. High Energy Phys. 08 (2009) 030.

[21] A. Collinucci, J. High Energy Phys. 04 (2010) 076.

[22] R. Blumenhagen, T. W. Grimm, B. Jurke, and T. Weigand, J. High Energy Phys. 09 (2009) 053.

[23] J. Marsano, N. Saulina, and S. Schafer-Nameki, J. High Energy Phys. 08 (2009) 046.

[24] R. Blumenhagen, T. W. Grimm, B. Jurke, and T. Weigand, Nucl. Phys. B829, 325 (2010).

[25] J. Marsano, N. Saulina, and S. Schafer-Nameki, J. High Energy Phys. 04 (2010) 095.

[26] T. W. Grimm, S. Krause, and T. Weigand, J. High Energy Phys. 07 (2010) 037.

[27] M. Cvetic, I. Garcia-Etxebarria, and J. Halverson, J. High Energy Phys. 01 (2011) 073.

[28] C. M. Chen, J. Knapp, M. Kreuzer, and C. Mayrhofer, J. High Energy Phys. 10 (2010) 057.

[29] C. M. Chen and Y. C. Chung, J. High Energy Phys. 03 (2011) 049.

[30] T. W. Grimm and T. Weigand, Phys. Rev. D 82, 086009 (2010).

[31] J. Knapp, M. Kreuzer, C. Mayrhofer, and N. O. Walliser, J. High Energy Phys. 03 (2011) 138.

[32] M. J. Dolan, J. Marsano, N. Saulina, and S. SchaferNameki, Phys. Rev. D 84, 066008 (2011).

[33] J. Marsano and S. SchaferNameki, J. High Energy Phys. 11 (2011) 098.

[34] T. W. Grimm, M. Kerstan, E. Palti, and T. Weigand, J. High Energy Phys. 12 (2011) 004.

[35] D. R. Morrison and D. S. Park, J. High Energy Phys. 10 (2012) 128. 
[36] C. Mayrhofer, E. Palti, and T. Weigand, J. High Energy Phys. 03 (2013) 098.

[37] V. Braun, T. W. Grimm, and J. Keitel, J. High Energy Phys. 09 (2013) 154.

[38] J. Borchmann, C. Mayrhofer, E. Palti, and T. Weigand, Phys. Rev. D 88, 046005 (2013).

[39] M. Cvetic, D. Klevers, and H. Piragua, J. High Energy Phys. 06 (2013) 067.

[40] V. Braun, T. W. Grimm, and J. Keitel, J. High Energy Phys. 12 (2013) 069.

[41] M. Cvetic, A. Grassi, D. Klevers, and H. Piragua, J. High Energy Phys. 04 (2014) 010.

[42] M. Cvetič, D. Klevers, and H. Piragua, J. High Energy Phys. 12 (2013) 056.

[43] J. Borchmann, C. Mayrhofer, E. Palti, and T. Weigand, Nucl. Phys. B882, 1 (2014).

[44] M. Cvetic, D. Klevers, H. Piragua, and P. Song, J. High Energy Phys. 03 (2014) 021.

[45] I. Antoniadis and G. K. Leontaris, Phys. Lett. B 735, 226 (2014).

[46] C. Lawrie, S. Schafer-Nameki, and J. M. Wong, J. High Energy Phys. 09 (2015) 144.

[47] M. Cvetic, D. Klevers, H. Piragua, and W. Taylor, J. High Energy Phys. 11 (2015) 204.

[48] M. Cvetic, A. Grassi, D. Klevers, M. Poretschkin, and P. Song, J. High Energy Phys. 04 (2016) 041.

[49] Y. Kimura and S. Mizoguchi, Prog. Theor. Exp. Phys. 2018, 043B05 (2018).

[50] Y. Kimura, J. High Energy Phys. 05 (2018) 048.

[51] M. R. Gaberdiel, T. Hauer, and B. Zwiebach, Nucl. Phys. B525, 117 (1998).

[52] O. DeWolfe and B. Zwiebach, Nucl. Phys. B541, 509 (1999).
[53] O. DeWolfe, T. Hauer, A. Iqbal, and B. Zwiebach, Adv. Theor. Math. Phys. 3, 1785 (1999).

[54] J. H. Schwarz, Phys. Lett. B 360, 13 (1995); 364, 252(E) (1995).

[55] T. Tani, Nucl. Phys. B602, 434 (2001).

[56] L. Bonora and R. Savelli, J. High Energy Phys. 11 (2010) 025.

[57] M. Fukae, Y. Yamada, and S.-K. Yang, Nucl. Phys. B572, 71 (2000).

[58] A. Grassi, J. Halverson, and J. L. Shaneson, J. High Energy Phys. 10 (2013) 205.

[59] A. Grassi, J. Halverson, and J. L. Shaneson, Commun. Math. Phys. 336, 1231 (2015).

[60] A. Grothendieck, Esquisse d'un Programme, (1984 manuscript), in Schneps and Lochak, London Mathematical Society Lecture Note Series (Cambridge University Press, Cambridge, England, 1997), Vol. I, pp. 5-48. English translation, pp. 243-283. MR1483107.

[61] S. K. Lando and A. K. Zvonkin, Graphs on Surfaces and Their Applications, Encyclopaedia of Mathematical Sciences: Lower-Dimensional Topology II (SpringerVerlag, Berlin, New York, 2004), Vol. 141.

[62] N. Seiberg and E. Witten, Nucl. Phys. B431, 484 (1994).

[63] A. Sen, Nucl. Phys. B475, 562 (1996).

[64] M. Bershadsky, K. A. Intriligator, S. Kachru, D. R. Morrison, V. Sadov, and C. Vafa, Nucl. Phys. B481, 215 (1996).

[65] D. R. Morrison and W. Taylor, J. High Energy Phys. 01 (2012) 022.

[66] S. Mizoguchi, J. High Energy Phys. 07 (2014) 018.

[67] S. Mizoguchi and T. Tani, Prog. Theor. Exp. Phys. 2016, 073B05 (2016). 